\title{
A CASE OF SCLEROTIC ATROPHY OF CEREBRUM AND CEREBELLUM, OF FAMILIAL TYPE, OCCURRING IN A BOY.
}

\author{
BY J. MICHELL CLARKE, M.A., M.D.CANTAB., F.R.C.P. \\ Professor of Pathology, Universzty College, Bristol; Physician to Bristol \\ General Hospital.
}

Arthun B., aged $7 \frac{1}{2}$ years, was admitted into the Bristol General Hospital on June 29, 1900. The grandmother (mother's mother) was blind until 11 years of age, and then recovered.

The mother said that she had two brothers, both younger than herself; the elder was quite well up to 10 years of age, when he became paralysed, went blind, and died of "lockjaw," aged 12. The second was also well until about 10 , when he gradually became paralysed and blind, and died at 13. No more details could be obtained of these patients, and the mother, on enquiry, could learn of no other similar cases amongst her relations. She was an intelligent woman, and was positive that the illness in her brothers' cases presented the same features as in her son. The patient had three brothers, all of them alive and well. The mother had had no miscarriages, and there was no history of syphilis in either parent. The father seemed a steady man, but neither he nor the mother appeared strong, and were rather excitable and of neurotic type.

The boy was quite strong and healthy until 7 years of age, and had never had any bad illness. The first symptoms noticed were that he did not care to go out, and he had slight choreiform jerkings of the arms and legs. The schoolmistress noticed that his hand jumped in writing. His sight gradually failed, and is now very defective, the right eye being especially defective. Eight weeks ago he began to vomit in the morning, but this has now stopped. The face had been drawn to one side. He said queer things, has been very irritable, easily losing his temper with his mother, and his speech has become indistinct lately. The headache has been on the top of the head. He has had no fits, no affection of bladder or rectum; he has been decidedly 
weak on his legs, easily falls down, and at times will walk right into a wall without observing it. No discharge from ears. He was admitted into the Bristol Hospital on June 29, 1900 . He seemed an intelligent boy, and answered questions sensibly. His speech was slow, with intervals between the words, which were also muffled and indistinct. He could sit up in bed, and could walk, but not well. The movements of the legs were uncertain or somewhat ataxic, especially of the right leg, so that he was unsteady, and he also tended to turn and to walk to the left. When walking there were jerkings of the arms, especially of the right arm, and when at rest jerkings, choreiform in character, of both arms and legs occurred. Even at this period, when standing he tended to fall backwards. He could not see to read, but had been able to read before the illness.

The boy was rather thin and badly nourished. His face had an old look. The thoracic and abdominal organs, and condition of the urine, were all normal.

There was no facial paralysis, no oculomotor paralysis, no nystegmus. The pupils were equal, normal in size, and acted well to light and accommodation. Sight extremely defective; he could just count fingers; no change present in optic disc, yellow spot region, or rest of fundus. (This was confirmed by Mr. Walker, Ophthalmic Surgeon to the Hospital.) Hearing normal. No actual paralysis anywhere at this date, but right leg weaker than left, and slight rigidity in each. No muscular wasting. No affection of sensation anywhere to touch or pain. Swallowing normal. Micturition and defæcation normal. No incontinence. Knee-jerks much exaggerated; plantar reflexes active; Babinski's sign present. No ankle clonus.

There was no pain or tenderness over the head or spinal column, and no deformity of them, nor of any other part. No club foot. He left the hospital after two days, as he cried perpetually to go home again and refused his food. During the time he stayed in he appeared to have a good deal of pain in the hesd. Subsequently he attended as an out-patient.

On August 9 he was decidedly worse; his sight was worse, and he was losing flesh. His mental condition was far worse; he had difficulty in understanding what was said to him, while before he would have understood at once; his memory was bad, and he was much more ohildish; he would ask his mother for things that he was holding in bis hands, and a fow minutes after she had given them to him be would forget that he had had them. At times he was very excited. His speech was more indistinct. 
He was now apparently a little deaf, but it was difficult to be sure of this on account of the mental dulness. He kept his mouth open, and bis lower lip dropped. Otherwise there was no facial or oculomotor palsy; no nystagmus; pupils normal. There were still slight irregular jerkings of limbs, but not so marked. Ho walked with an uncertain, ataxic gait, weak and unsteady, and hod a tendency to fall backwards. He was never still, but kept walking backwards and forwards. Both legs were rigid, with exaggerated knee-jerks, and with extension of toes in plantar reflexes, but no ankle-clonus. The great toes were in constant position of hyper-extension. There was now a tendency for him to wet his bed.

In September there was incontinence of urine; be passed his frees into his clothes, and was unable to feed himself.

On October 25.-Condition worse. His sight had been steadily failing, and be was now quite blind, and remained so. At the same time the optic discs and fundus oculi remained normal, and the pupils reacted to light (feebly). The pupils were now large, and slightly unequal. Mentally be was more dull, but happy, laughing and chuckling to himself; he recognised a penny, knew my name, and put out his tongue when commanded, but this was about the extent of his powers. His mouth was open, saliva dribbling from it. He could just stand alone, balancing himself with his feet wide apart, and falling backwards at the least touch; he could only walk with support, taking short steps with bis feet wide apart. He could not sit up alone, falling backwards. The knee-jerks were greatly exaggerated, there was slight ankle clonus, and marked rigidity of both legs.

In November he had an attack of herpes zoster, affecting the fourth and fifth left intercostal spaces; this was accompanied and followed by much pain. He was quite blind, and at times cried out with pains in the head; there was no vomiting. He could swallow fairly well; his speech had become very indistinct, and except when he cried out for food or because of pain, he spoke very little. The cranial nerves were unaffected. He was unable to feed himself. He had somewhat more control over the sphincters than in the previous month. He could just stand, but tended to fall backwards, and when he tried to walk he went backwards until he fell. The arms were now rigid, with exaggerated deep reflexes; spastic rigidity in the legs was very marked, with ankle and knee clonus on each side.

In December he had lost the power of standing or walking unsupported; when placed upon his feet he fell backwards at once. 
His speech was very slurred and indistinct, saliva constantly dribbled from his mouth, and during sleep he made munching movements. Both arms and legs were the seat of marked spastic contracture, and were affected, the legs especially, with painful cramps.

In January he had an access of severe pains in the head and of painful cramps in the legs, and apparently in the abdominal muscles. He also suffered from obstinate constipation. He could not sleep, and cried out day and night, so that he was admitted again into the hospital. He was now quite bed-ridden, helpless, and it was impossible to make him understand anything. Both arms and legs were rigidly contracted, the latter being flexed at the hips and knees and drawn up upon the abdomen. There was no affection of sensation. The pupils were moderately dilated; there was no change in the optic discs. He was much wasted, had incontinence of urine; there were no bed-sores. He cried out constantly for his mother, and would take food from no one else, so that she took him out of the hospital the next day. $\mathrm{He}$ died shortly afterwards, constantly crying out or screaming some days before desth.

Permission was given after some difficulty to examine the bead only, and this examination was made in a slum under very adverse conditions, and I could only take away with me small portions of the brain, and not the whole of it, as I should have wished.

I could not get leave to examine the cord. This must be my excuse for the imperfections of the pathological report.

On examination the brain was small, the dura mater healthy, the pia-arachnoid was not decidedly thickened, but its vessels were in places injected, especially over the posterior part of the brain. There was no evidence of past or present meningtis. The cerebro-spinal fluid was present in excess. The cerebral cortex was somewhat injected. The brain was normally developed, and there was no obvious change in the arrangement of the convolutions, but those of the occipital lobes and motor area were small. As a whole the posterior pole of the hewispheres was unduly small. The optic nerves and tracts, the cranial nerves generally, and corpora quadrigemina appeared healthy.

On cutting into the cerebral hemispheres, the chief 
naked-eye changes were a patch of recent softening in the left internal capsule, involving the greater part of it, and a tough, indurated or sclerotic condition of the white matter of the occipital lobes, which was grey, dense, hard and firm. The occipital cortex was paler and thinner than normal, and this was true in less degree of the motor cortex. The white matter of the ascending frontal and parietal convolutions was firmer, and the right internal capsule greyer in colour than normal. The basal ganglia and subthalamic regions appeared bealthy. The ventricles were somewhat dilated, their ependyma healthy.

The cerebellum was rather small; externally it had a healthy appearance; the cortex looked normal on section, but the white matter showed an even greater sclerotic change than that of the occipital lobe, being dense, hard, tough, grey in colour, and cutting like tough fibrous tissue, almost creaking under the knife.

No new growth was anywhere found, nor any sign of a gummatous formation. The bones of the skull were normal, and the calvarium not undaly thickened. The vessels at the base were healthy, and contained no clot. They had the normal arrangement.

The bones at the base and the tympani were normal. Portions from the cerebellum, both occipital lobes-onter surface and in region of calcarine fissure-motor convolutions, and præfrontal areas on each side, optic nerves, region of internal capsules, crura cerebri, pons, medulla, and upper end of cervical cord, were examined microscopically.

Nissl's stain, Pal-Weigert, with carmine, Marchi's method, Van Gieson's stain, logwood and eosin, were used for cortex cerebri and cerebellam; and for pons and medulla, PalWeigert and carmine, and Marchi's method.

In the cerebram, the most pronounced changes were found in the occipital cortex, and these were by far most intense in the neighbourhood of the calcarine fissures. The pia-arachnoid, under the microscope, appeared slightly injected, and the larger vessels distended. The superficial layer of cortex, and the first and second layers of granule 
cells, appeared healthy. The whole cortex was narrowed, and the pyramidal cells were in all stages of degeneration and atropby. The large pyramids and deepest layer of cortex were most affected; next the small pyramids.

The changes were not uniform, but patchy in distribution. There were numerous small spaces in the grey matter (where atrophied cells had disappeared?). The vessels showed \& moderate degree of injection, the neuroglial cells were increased in number, and in the parts in which the destructive process was most advanced there was a round-celled infiltration from the vessels, and phagocytes could be seen attached to the pyramidal cells in some instances. Vascular changes were more marked in the layer of white matter immediately subjacent to the grey; here many vessels were crowded with leucocytes, which were passing in numbers into the perivascular space and surrounding tissues. In the white matter, in very few places in the grey, there is to be seen extremely abundant proliferation of the endothelial cells lining the perivascular space, so that the latter was crammed with large endothelial plates with distinct small. round nuclei. The vessel walls themselves were mostly normal, but some showed slight thickening. There was no sign in any of endarteritis.

In the white matter of this lobe, extensive areas were fond in which, with the above vascular changes, the tissue consisted of a dense network of spider cells, with large, richly-branched, and coarse processes, forming an almost myxomatous-like tissue, but obviously composed of the greatly-proliferated neuroglial cells of the part. In these aress the white fibres bad practically disappeared, and in others in this lobe, with less advanced change, were few in number. Sections did not stain in Marchi's fluid, but by Pal-Weigert stain the fine tangential fibres of the cortex were greatly reduced in number, in places almost absent, and in the deeper parts of the white matter there were areas in which the medullated fibres passing from convolutions were degenerated.

In sections from the motor convolutions there were similar changes, but less marked; the distribution was more 
partial and less intense in degree. In the cortex, the large pyramidal cells and the multipolar cells of the lowest layer were chiefly affected, there being a considerable number of cells in different stages of degeneration in these layers; on the other hand, there were numerous healthy cells. To a less extent the layer of small pyramidal cells was also affected. The capillaries were injected in places, with emigration of leucocytes and increase of nearoglial cells (Nissl stain).

The vascular changes in white matter were slight, and there was an absence of proliferation of endothelium of lymph spaces. $A_{8}$ in the occipital cortex, the underlying white substance was most affected in those places where the changes in the grey matter were most evident. In the most affected areas the tangential fibres of cortex appeared deficient in number (Pal-Weigert).

Sections from the pro-frontal area showed that the grey and white matter were practically normal, with the exception that there were a few scattered pyramidal cells in which the protoplasm had a swollen, glassy appearance, the Nissl granules were absent, and the nucleus obscured, or excentric in position, and there were also a very few atrophied pyramidal cells. By Marchi's method, sections from cerebrum stained badly, and failed to give any additional information.

Cerebellum (Nissl stain).-Granule layer normal, and at first sight Pinkinje's cells normal, bot on examining several sections it became probable that they were really somewhat deficient in number when compared with the normal cerebellum. Although the vast majority of them had a healthy appearance, some were rounded in shape, had lost their processes, stained deeply and aniformly, so that the granules could not be distinguished, and nuclei were obscured.

(Pal-Weigert). - In small folia, fine fibres of white matter stained normally, but in tracing them down they passed in several instances into patches, in which they disappeared (sclerotic areas ?), to emerge again in greatly reduced numbers. The fibres could not in any case (probably from change in direction) be traced a great distance, but in the larger stems, just above the point beyond which they could 
not be traced, the medullated fibres sometimes became tortuous and separated by wider intervals.

(Marchi).-By this method, sparsely-scattered degenerated fibres were found in all sections; most abundant in small, but traced in some cases into medium-sized or large stems of folia.

In layer of white matter subjacent to grey cortex, could be seen in many places injection of vessels and infiltration with leucocytes, and in the larger stems of the white matter there was, in addition, increase of the interstitial tissue. (Van Gieson and hæmatoxylin and eosin stains.)

These changes affected middle and both lateral lobes. On the whole the pathological changes in the cerebellum were not so marked under the microscope as the very striking naked-eye changes had led one to expect. In the crura, cerebri, pons, medulla, and beginning of cervical cord, sections stained by Pal-Weigert showed a complete and absolute degeneration of the pyramidal tract on each side no healthy fibres appeared to be left; bat by Marchi's method each pyramid was found to contain 20 to 30 fibres which took this stain, and had presumably degenerated more recently.

In the pons (Marchi's stain) there were found a few scattered degenerated fibres in the lateral and especially in the mesial fillet, on each side, and a few also in each superior cerebellar peduncle; but these were only few, the vast majority of fibres being healthy. In sections throngh the medulla there were also a few scattered degenerated fibres in the restiform body on each side, a very few in the reticular formation, in the issuing left sixtb nerve-root, and in the peduncle of the left flocculus cerebelli.

I much regret that the post-mortem examination was unavoidably so imperfect. and that I was not able to obtain the cord. From the sections at the decussation of the pyramids, and immediately below it in the cervical cord, it appears plain that the degenerations were entirely descending, and that there were no ascending ones.

To sum up briefly the whole case : Clinically the disease was characterised by appearing without ascertainable cause VOL. XXV. 
in a previously healthy boy of 7, two of his mother's brothers having died from a disease having like symptoms and running a similar course, at the ages of 12 and 13 respectively (and his grandmother heving been blind in childhood and recovered). The first symptoms were choreiform jerkings of limbs and failure of sight, going on to ataxy of cerebellar type, blindness, without affection of lower visual segment or optic neuritis, indistinct speech, increased tendon-reflexes and spastic rigidity, a kind of forced movements, constant tendency to fall on the back of his head, with gradually increasing mental dulness, ending in paralysis of all limbs, spastic painful contractures, progressive dementia, emaciation, and deatb from exhaustion.

Pathologically, so far as was ascertained, on both sides of the brain there was destruction, atrophy of cortical neurones in grey matter, and sclerosis of white matter of occipital lobe, most marked on mesial aspect (calcarine fissure); the same changes, but to a less degree, in motor convolutions, yellow softening in left internal capsule, complete degeneration of pyramidal tracts on both sides, sclerosis of white matter of cerebellum (both sides), and a few scattered degenerated fibres in fillets, superior cerebellar peduncle and restiform body.

A few general considerations as to the nature of this case may not be out of place. In many respects it corresponds to some of the cases deslt with by Dr. Collier in his valuable paper on cerebral diplegia, ${ }^{1}$ and especially to those examples of cerebral diplegia of post-natal origin with generalised cerebral rigidity, but differs from them by the addition of cerebellar symptoms and peculiar form of blindness. The first point to be settled is the question whether the history is sufficiently definite to warrant the view that the case belongs to a "family" disease. As the other cases were not seen, there is only the mother's statement to go upon, and with regard to that it can only be said that she was an intelligent woman, and that the symptoms were sufficiently distinctive to avoid confusion. One could not, however, say that the blindness was due to exactly the same

'Brais, vol. xxil., 1899. 
cause as in the present case, as the other patients may have had cerebral diplegia, with the optic atrophy that not uncommonly accompanies it. Admitting, however, that the three cases occurring in two generations of the family, with, at any rate, a striking correspondence in symptoms and age at onset, justify one in regarding them as belonging to the familial class of nervous disease, then the present case may be assigned to a group of more or less similar diseases of the brain occurring in childhood or adolescence, and presenting considerable diversity in range of clinical symptoms in individual instances. The age at onset is important, as, in order to avoid complication by the inclusion of congenital defects or diseases of the brain, or injuries arising in various ways during parturition, it is better, provisionally, at all events, to exclude cases in which the first symptoms of disease are noted in infancy.

By thus narrowing the field of observation, and further restricting it to familial diseases, the affections which stand in nearest relation to the case above reported are (1) certain cases of cerebral diplegia beginning in childhood, and (2) probably some cases of family spastic paraplegia. Taking the latter first, in the series of twenty-nine cases collected by Lorrain ${ }^{1}$ the average age of onset was 8 to 15 years. No pathological changes are given, but the author draws the important conclusion from his cases that the family diseases of the nervous system are not sharply defined from each other, but are connected by transitional forms. In two cases reported by Mdlle. Pesker, ${ }^{2}$ of two brothers, we find such a transitional form, for, in addition to spastic paraplegia, there was intention-tremor and ataxia in the arms, affection of speech, and decided mental weakness (in Lorrain's cases the mind was unaffected) ; and in the case of one brother, who died, besides changes in the posterior columns and Clarke's columns in the cord, there was deficiency in nerve-fibres and in development of ganglion-cells in the cerebral cortex.

Cerebral diplegia, although rarely, does occur in familial form, and many cases are now recorded. ${ }^{8} \quad$ The symptoms in

1 Thess do Paris, 1898.

2 Revtwe Neuralogique, vol. viii., p. 849.

" See "Deroum."-J. of Nerv. and Mental Dis., 1897, p. 898-9, where soveral references are giveu. 
cerebral diplegia, in the particular form now under consideration, may be pat very briefly as general muscular rigidity, spastic contractures of limbs, exaggerated deep reflexes, more or less paralysis, tremors, affection of speech, and often choreiform or athetotic movements, optic atrophy, with more or less defect of intelligence. The wental affection varies very greatly, in some cases being slight until towards the end of the illness, in others being marked throughout, like those reported by Homen, ${ }^{1}$ where, with similar bodily symptoms to those just detailed, the mental changes were so pronounced as to make the cases resemble juvenile general paralysis. It is true that in these particular cases the cause was probably congenital syphilis. The course of the disease is progressive, the duration varying.

The symptoms in particular cases will vary according to the part of the brain on which the chief stress of the disease falls. In the majority of cases, this will be on the motor convolutions, with more or less accompenying mental changes, according to the degree of affection of the prefrontal area. In Homen's cases just quoted, the chief changes were found in the frontal lobes.

The peculiar point which would seem to make the case I have reported a very exceptional one is that the cerebellum was also affected, and consequently cerebellar symptoms were prominent through a large part of the course of the disease, and in addition, the changes in the cerebrum being most pronounced in the occipital lobe, there was the striking symptom of blindness without any change in the eye itself or in the optic nerve.

I have not been able to find this symptom recorded in any other case in the limited search I have made into the literature, bat I have not attempted an exhaustive one. Iu Dr. Collier's paper, ${ }^{8}$ he quotes Richardière as saying that the atrophy in cerebral diplegia has a special preference for the occipital lobe in many cases, and he also says that in connection with atrophy of the hemispheres there may be marked atrophy of the cerebellum.

'Neurol. Centlbltt., 1897, p. 864.

= Lac. cit. 
The cases that approach most nearly to mine in clinical symptoms are the following :-

Drs. Pauly and Bonné ${ }^{1}$ have pablished an account of a family in which three sons suffered from the same nervous affection; two others were stillborn, and five daughters were healtby. The chief symptoms were spastic condition of legs, intention-tremor, speech-disturbances, optic atrophy, nystagmus, spastic, staggering gait, with absence of affection of sensation of the sphincters or of intelligence. Deep reflexes exaggerated to clonus. The disease began in the eldest patient with staggering, in the second with spastic gait, and in the youngest with nystagmus. The authors conclude that their cases do not belong to a family form of insular sclerosis, nor to bereditary cerebellar ataxy, nor to family cerebral sclerosis, and regard the clinical symptomcomplex as a transitional one between hereditary cerebellar atraxy and cerebral diplegir.

I have also seen a short abstract of a paper by Cestan and Guillain, ${ }^{8}$ in which two cases of family spastic sclerosis are described. The patients were two brothers; one of them had always had an abnormal gait, and at the age of 15 speech disturbances appeared; some months later the gait was strikingly "spastic cerebellar."

With regard to the pathological changes in my case, their nature is not a specific one; in other cases more or less similar changes have been found. Their distribution, however, is unusual in its wide extent, in affection of cerebellum, and absence of implication of frontal lobe. In respect of the cerebellum it is certainly worthy of remark that the microscopic changes in the cerebellar cortex were so insignificant, when one bears in mind the marked cerebellor symptoms, and also the advanced degree of sclerosis of the white matter at the base or stems of the lobes.

The cause of the disease was not congenital syphilis; the history was absolutely decisive on this point. Although the proliferation of endothelium in perivascular spaces is seen in syphilis, it also occurs ander other conditions, and the absence 
of any change in the arteries themselves is very strongly against it, setting aside the absence of any gross syphilitic lesions.

In the absence of syphilis, an early form of general paralysis is extremely unlikely, and the distribution of the changes in the brain, and especially the escape of the frontal lobes, is not that of general paralysis, although the vascular and perivascular changes, in combination with the atrophy of the cortex, present a resemblance to it.

The microscopic appearances also differ from those found in most cases of cerebral diplegia ${ }^{1}$ in the more pronounced vascular changes and sclerosis of white matter of the affected lobes, the essential change in these cases being the atrophy of the cortical neurons. As in most cases of this kind, it is extremely difficult to decide whether the change is a primary one in the cortical neurons, and the sclerotic and vascular changes merely secondary to the disappearance of the parenchymatous elements, or whether on the other hand the disease is due to a poison circulating in the blood, the alterations in the vascular system being first produced, and the destruction of the cortical neurons secondary to them.

It is well to bear in mind that the sclerotic process with vascular and perivascular changes was more prononnced in the white than in the grey matter in this case. It is, T. suppose, conceivable that even when the primary change is in the neurons, the amount of secondary sclerosis and vasculax change may vary very much in intensity in different cases, at one end of the scale being very slight, at the other constituting a predominant feature of the pathological alterations.

In favour of the view that the condition here is primary in the neurons, is the character of the onset-insidions, without fever or other constitutional disturbance, not preceded by one of the specific fevers, all points unlike the action of a toxin. The course of the disease, though not long compared with that of some other family diseases, is more consonant with this view, and there is the evidence of other cases in the family. The fact, also, that in this case

\footnotetext{
1 Bee Collis, loc. cit.
} 


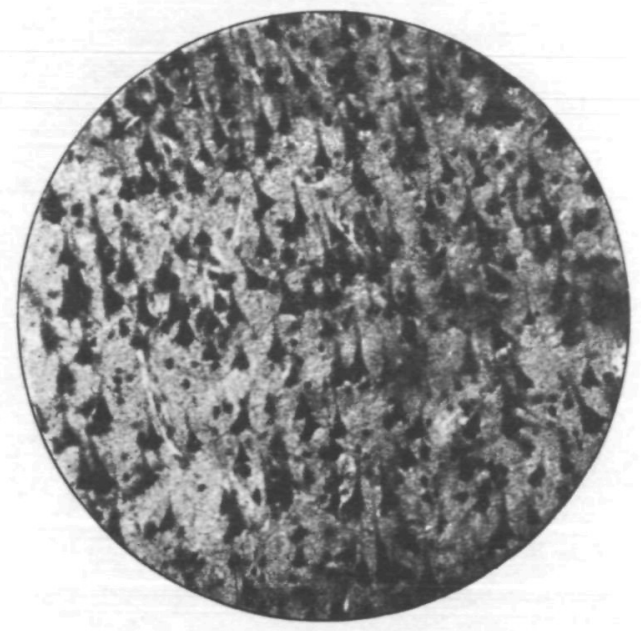

Fir. 1

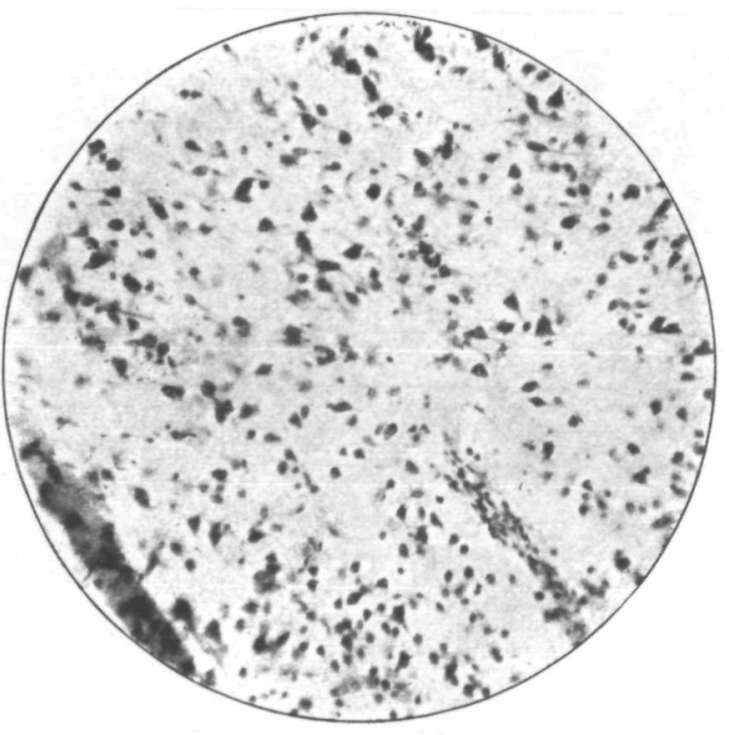

FIg. 2. 


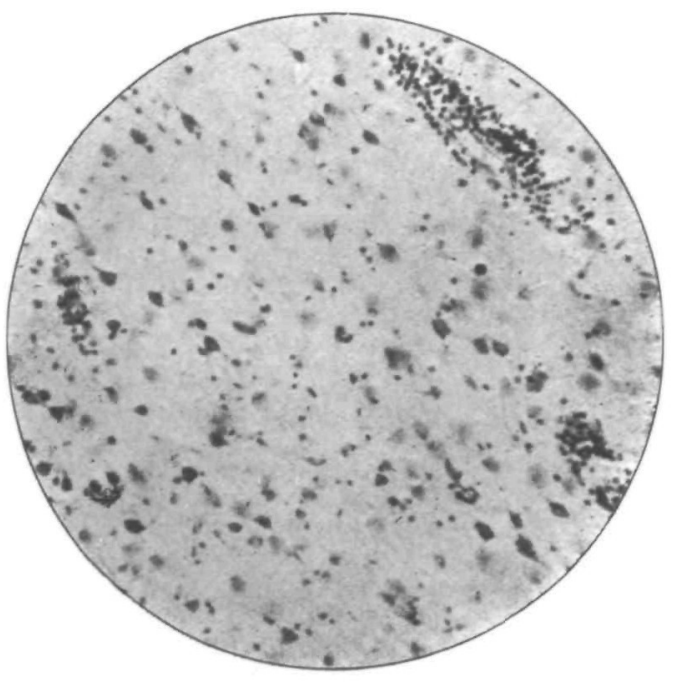

FIG. 3.

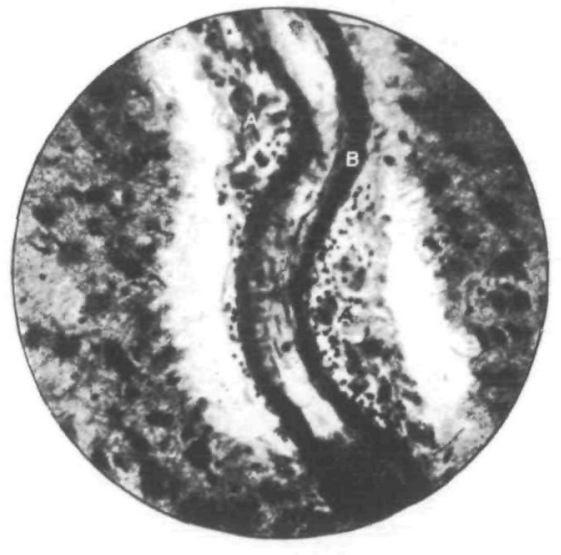

FIG, 4

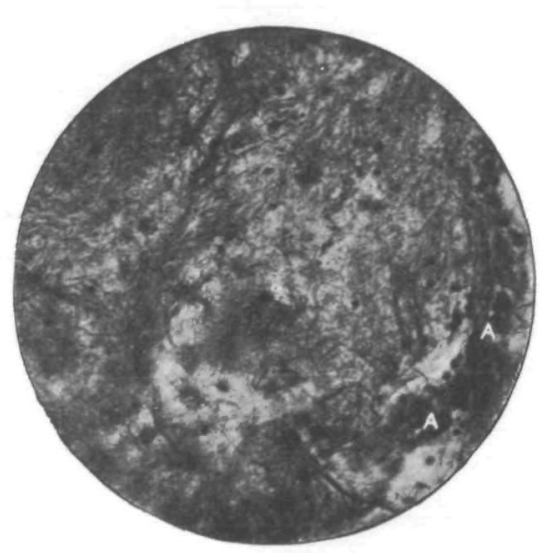

FiG. 5. 


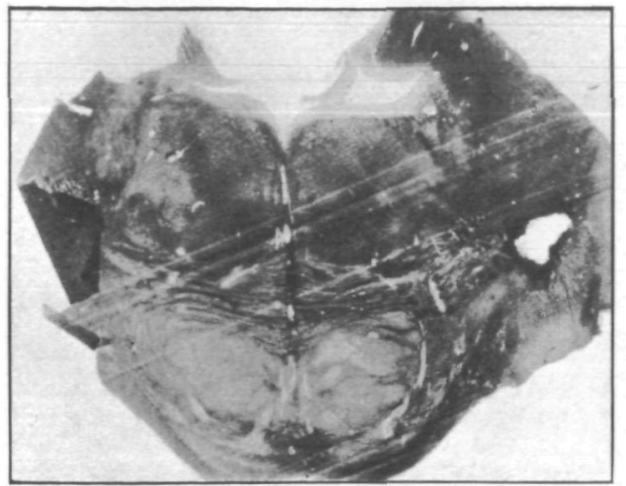

Fig. 6.

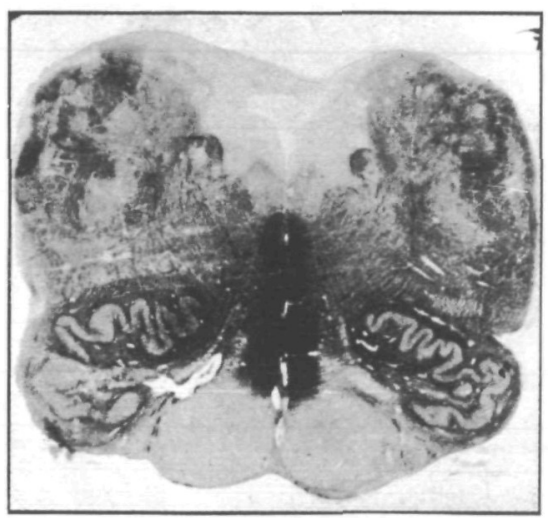

FIG, 8.

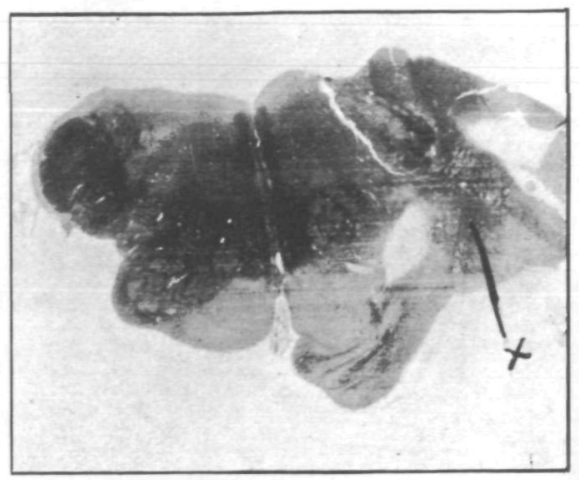

FIG. 7.

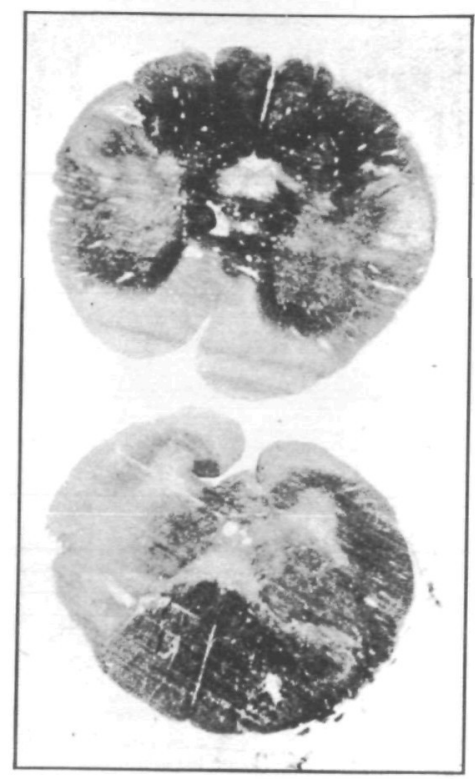

Fig. 9. 
. 
the disease appeared at an age some years earlier than in the preceding generation, tells in the same direction. The distribution of the disease in the brain is difficalt to explain in any other way, and in connection with this it is to be noted that though vascular changes predominate in the white matter, there is a close correspondence between the affected weas of grey and white substance, those of the latter subjacent to the most altered portions of grey matter showing the most advanced changes.

Perbaps the above case may be best described as clinically intermediate between cerebral diplegia of postnatal origin and hereditary cerebellar ataxy, with the occurrence of the peculiar form of blindness due to the special incidence of the disease upon the occipital lobes. As similar types of disease appear to reproduce themselves with some fidelity, this peculiar combination of clinical symptoms may again be met with.

My best thanks are due to Mr. James Taylor and Mr. Moline for kindly taking the photographs for me.

\section{EXPLANATION OF PHOTO-MICROGRAPHS.}

Fia. 1.-Section of cortex of profrontal area, apparently normal, for comparison with

F10. 2.-Section of ocoipital cortex, showing advanced degeneration and atrophy of pyramidal cells. A vessel at left-hand margin shows proliferation of endothelial cells of perivascular space, and another further to the right (at lower margin) shows infltration of round cells. Two or three pyramidal cells can be seen which have retained their normal shape.

FIa. 3.-Section through junction of gray and whito mattar. The latter (right half of figure) shows round.celled infiltration around vessels in subjacent layer of white matter. Left half of figure shows degenerated cortex.

The above three sections are stained by Nisal's method; the magnification is about 180 , in order to get a fair-sized field. Figs. 4 and 5 are stained with logwood and picro-fuchsin.

Fia. 4 shows an artery from sclerosed white matter of acoipital lobe. The wall of the artery itself is normal, but on each side of it, in perivasoular space, is a mass of cells, chiefly composed of large endothelial cells from luing of space, with some leucocytes amongst them. The dark cells in the part of the fleld to the left of the ertery are spider-cells.

f'Ia. 5 shows the network of richly-branched cells, which make up the desse sclerotic aress, and to the right, marked AA, is a small vessel almost lidden by the proliferation of the endothelium of perivascular space. This suction is under a higher magnification.

Fias. 6, 7, 8 and 9 are sections from various levels of pons, medulla, and at decussation of pyramids, which are stained by the Pal. Weigert method, tud show absolute and complete degeneration of both pyramidal tracts. At $x$ in Fig. 7 is a small tract of degenerated flbres in the peduncle of the Hocculus. 